\title{
HAT-P-10b: A LIGHT AND MODERATELY HOT JUPITER TRANSITING A K DWARF*
}

\author{
G. Á. Bakos ${ }^{1,9}$, A. PÁL ${ }^{1,2}$, G. Torres ${ }^{1}$, B. Sipő́cz ${ }^{1,2}$, D. W. Latham $^{1}$, R. W. Noyes ${ }^{1}$, Géza Kovács ${ }^{3}$, J. Hartman ${ }^{1}$,

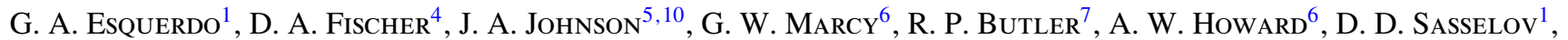 \\ GÁbor Kovács ${ }^{1}$, R. P. StefaniK ${ }^{1}$, J. LÁZÁR ${ }^{8}$, I. PAPP ${ }^{8}$, AND P. SÁRI ${ }^{8}$

\section{ABSTRACT}

We report on the discovery of HAT-P-10b, one of the lowest mass $\left(0.487 \pm 0.018 M_{\mathrm{J}}\right)$ transiting extrasolar planets (TEPs) discovered to date by transit searches. HAT-P-10b orbits the moderately bright $V=11.89 \mathrm{~K}$ dwarf GSC 02340-01714, with a period $P=3.7224747 \pm 0.0000065$ days, transit epoch $T_{c}=2454759.68683 \pm 0.00016$ (BJD), and duration $0.1090 \pm 0.0008$ days. HAT-P-10b has a radius of $1.005_{-0.027}^{+0.032} R_{\mathrm{J}}$ yielding a mean density of $0.594 \pm 0.052 \mathrm{~g} \mathrm{~cm}^{-3}$. Comparing these observations with recent theoretical models we find that HAT-P$10 \mathrm{~b}$ is consistent with a $\sim 4.5 \mathrm{Gyr}$, almost pure hydrogen and helium gas giant planet with a $10 M_{\oplus}$ core. With an equilibrium temperature of $T_{\mathrm{eq}}=1020 \pm 17 \mathrm{~K}$, HAT-P-10b is one of the coldest TEPs. Curiously, its Safronov number $\theta=0.053 \pm 0.002$ falls close to the dividing line between the two suggested TEP populations.

Key words: planetary systems - stars: individual (HAT-P-10, GSC 02340-01714) - techniques: photometric techniques: spectroscopic

Online-only material: machine-readable table

\section{INTRODUCTION}

It has become clear in recent years that transiting extrasolar planets (TEPs), especially those around bright stars, are extremely valuable for understanding the physical properties of planetary bodies. The transit itself is a periodic event, whichtogether with high-precision spectroscopic observations and radial velocity (RV) follow-up-reveals a number of key parameters, notably the relative radius of the planet with respect to the star, and the true mass of the planet without the inclination ambiguity. These allow determination of the mean density of the planet, and an insight into its basic structural properties. These advantages have been realized early on, and the recent rise in the detection of TEPs is due to a number of dedicated transit searches, such as TrES (Brown \& Charbonneau 2000; Dunham et al. 2004), XO (McCullough et al. 2005), SuperWASP (Pollacco et al. 2006), OGLE (targeting fainter stars; Udalski et al. 2008), and HATNet (Bakos et al. 2002, 2004). At the time of this writing, the number of published TEPs with a unique identification is $\sim 40$, with $\sim 35$ of these due to dedicated transit searches. The properties of known TEPs already span a wide range, from the hot Neptune GJ436b with a mass of $M_{p}=0.072 M_{\mathrm{J}}$ (Butler et al. 2004; Gillon et al. 2007) to XO-3 with $M_{p}=11.79 M_{p}$ (Johns-Krull et al. 2008), from shortperiod orbits like OGLE-TR-56b with $P=1.2$ days (Udalski et al. 2002; Konacki et al. 2003) to $P=21.2$ days of HD $17156 \mathrm{~b}$

\footnotetext{
* Based in part on observations obtained at the W. M. Keck Observatory, which is operated by the University of California and the California Institute of Technology. Keck time has been granted by NOAO (A285Hr) and NASA (N128Hr).

9 NSF Fellow.

${ }^{10}$ NSF Postdoctoral Fellow.
}

(Barbieri et al. 2007). Although most of these planets have circular orbits, some planets with significant eccentricities, such as GJ436b, HAT-P-2b (Bakos et al. 2007a), XO-3b, or HD 17156b have also been reported. TEPs have been discovered in a wide range of environments, from orbiting $\mathrm{M}$ dwarfs (GJ436b) up to mid-F dwarfs, such as HAT-P-7b (Pál et al. 2008b).

Theoretical investigations have been thriving during this vigorous discovery era, some focusing on the radius of these planets (Burrows et al. 2007; Liu et al. 2008; Chabrier et al. 2004; Fortney et al. 2007) and others on the atmospheres (e.g., Burrows et al. 2006; Fortney et al. 2007), to mention two of the key observable properties of transiting planets. When confronting theory with observations, it is also essential to use accurate observational values, along with proper error estimates. The recent re-analysis of TEP parameters by Torres et al. (2008) represents a step forward in this sense. It was also noted throughout these works that a much larger sample is required for better understanding of the underlying physics, i.e., more planets are needed to populate the mass-radius (or other) parameter space, to improve the statistical significance of correlations between planetary and stellar parameters, and to reveal any previously undetected correlations that may shed light on the physical processes governing the formation and evolution of TEPs.

The HATNet survey has been a major contributor to TEP discoveries. Operational since 2003, it has covered approximately $7 \%$ of the Northern sky, searching for TEPs around bright stars $(8 \lesssim I \lesssim 12 \mathrm{mag})$. HATNet operates six wide field instruments: four at the Fred Lawrence Whipple Observatory (FLWO) in Arizona, and two on the roof of the Submillimeter Array Hangar (SMA) of SAO at Hawaii. Since 2006, HATNet has announced 


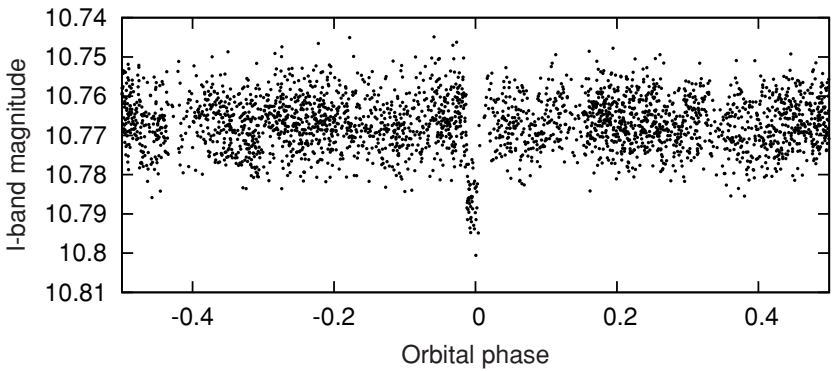

Figure 1. Unbinned light curve of HAT-P-10 including all 2870 instrumental $I$-band measurements obtained with the HAT-10 telescope of HATNet (see the text for details), and folded with a period of $P=3.7224747$ days (which is the result of the fit described in Section 4).

and published nine TEPs. In this work, we report on the 10th such discovery. ${ }^{11}$

\section{PHOTOMETRIC DETECTION}

The transits of HAT-P-10b were detected with one of the HATNet telescopes, HAT-10, located at FLWO. The region around GSC 02340-01714, a field internally labeled as G213, was observed on a nightly basis between 2005 October 3 and 2006 March 14, whenever weather conditions permitted. We gathered 2870 exposures of 5 minutes at a 5.5 minute cadence. Each image contained approximately 29,000 stars down to $I \sim 14.0$. For the brightest stars in the field, we achieved a per image photometric precision of 4 mmag.

The calibration of the HATNet frames was done utilizing standard procedures. The calibrated frames were then subjected to star detection and astrometry, as described in Pál \& Bakos (2006). Aperture photometry was performed on each image at the stellar centroids derived from the Two Micron All Sky Survey (2MASS) catalog (Cutri et al. 2003) and the individual astrometrical solutions. The resulting light curves were decorrelated against trends using the external parameter decorrelation technique (EPD; see Bakos et al. 2007b) and the trend filtering algorithm (TFA; see Kovács et al. 2005). The light curves were searched for periodic box-like signals using the box least-squares method (BLS; see Kovács et al. 2002). We detected a significant signal in the light curve of GSC 02340-01714 (also known as 2MASS 03092855+3040249; $\alpha=03^{\mathrm{h}} 09^{\mathrm{m}} 28^{\mathrm{s}} .55$, $\delta=+30^{\mathrm{d}} 40^{\mathrm{m}} 24.9$; J2000), with a depth of $\sim 15 \mathrm{mmag}$ and a period of $P=3.7225$ days. The dip had a relative duration (first to last contact) of $q \approx 0.027$, corresponding to a total duration of $P q \approx 2.5 \mathrm{hr}$ (see Figure 1).

\section{FOLLOW-UP OBSERVATIONS}

\subsection{Reconnaissance Spectroscopy}

All HATNet candidates are subjected to thorough investigation before using more precious time on large telescopes, such as Keck I, to observe them. One of the important tools for establishing whether the transit feature in the light curve of a candidate is due to astrophysical phenomena other than a planet transiting a star is the CfA Digital Speedometer (DS; Latham 1992), mounted on the FLWO $1.5 \mathrm{~m}$ telescope.

\footnotetext{
11 After submission of this paper, it was realized that HAT-P-10b and WASP- $11 \mathrm{~b}$ refer to the same object, independently discovered, with WASP-11b submitted 7 days earlier to A\&A (West et al. 2008). The two discovery groups agreed on calling it in future papers as WASP-11b/HAT-P-10b, with separate entries on www.exoplanet.eu.
}

Table 1

Relative RV Measurements of HAT-P-10

\begin{tabular}{lcccc}
\hline \hline $\begin{array}{l}\text { BJD } \\
(2,454,000+)\end{array}$ & $\begin{array}{c}\mathrm{RV}^{\mathrm{a}} \\
\left(\mathrm{m} \mathrm{s}^{-1}\right)\end{array}$ & $\begin{array}{c}\sigma_{\mathrm{RV}} \\
\left(\mathrm{m} \mathrm{s}^{-1}\right)\end{array}$ & $\begin{array}{c}\text { Bisector Span } \\
\left(\mathrm{m} \mathrm{s}^{-1}\right)\end{array}$ & $\begin{array}{c}\sigma_{\mathrm{BS}} \\
\left(\mathrm{m} \mathrm{s}^{-1}\right)\end{array}$ \\
\hline 547.76983 & 35.78 & 4.32 & $\ldots$ & $\ldots$ \\
548.75614 & 21.56 & 2.00 & -15.68 & 19.81 \\
675.11598 & 11.63 & 1.92 & -31.24 & 17.93 \\
723.07574 & 13.07 & 1.71 & 8.36 & 15.27 \\
725.08480 & 141.07 & 2.26 & 7.60 & 11.67 \\
726.96609 & $\ldots$ & $\ldots$ & 11.20 & 11.16 \\
727.13146 & 0.00 & 2.01 & 24.00 & 10.96 \\
806.81372 & 153.45 & 2.28 & 7.49 & 17.60 \\
806.81967 & 143.03 & 2.34 & -11.76 & 18.78 \\
\hline
\end{tabular}

Note. $\mathrm{BS}=$ bisector span.

${ }^{a}$ The fitted zero point that is on an arbitrary scale (denoted as $\gamma_{\text {Keck }}$ in Table 4) has not been subtracted from the velocities.

High-resolution spectra with low signal-to-noise ratio (S/ $\mathrm{N}$ ) from this facility have been used routinely in the past to derive RVs with moderate precision (roughly $1 \mathrm{~km} \mathrm{~s}^{-1}$ ) and to determine the effective temperature and surface gravity of the host star, to weed out false alarms, such as F dwarfs orbited by $\mathrm{M}$ dwarfs, grazing eclipsing binaries, triple and quadruple star systems, or giant stars where the transit signal is either false, or comes from a nearby, blended eclipsing binary.

The RV measurements of HAT-P-10 showed an rms residual of $0.43 \mathrm{~km} \mathrm{~s}^{-1}$, consistent with no detectable RV variation. Atmospheric parameters for the star, including the effective temperature $T_{\text {eff }}=5000 \mathrm{~K}$, surface gravity $\log g=$ 4.5 , and projected rotational velocity $v \sin i=1.5 \mathrm{~km} \mathrm{~s}^{-1}$, were derived as described by Torres et al. (2002). The effective temperature and surface gravity correspond to an early $\mathrm{K}$ dwarf.

\subsection{High-Resolution, High-S/N Spectroscopy}

Given the significant detection by HATNet, and the positive DS results that exclude the usual suspects, we proceeded with the follow-up of this candidate by obtaining high-resolution and high $\mathrm{S} / \mathrm{N}$ spectra to characterize the $\mathrm{RV}$ variations and to determine the stellar parameters with higher precision. We obtained eight exposures with an iodine cell, plus one iodinefree template, using the HIRES instrument (Vogt et al. 1994) on the Keck I telescope located on Mauna Kea, Hawaii. The observations were made on the nights of 2008 March 21-22, July 27, and on three nights between September 12 and 17. The small RV variations based on the March 2007 run made this target a firm planet candidate, but more observations were required to derive an orbit and to check spectral bisector variations (see Section 4.2).

The width of the spectrometer slit used on HIRES was 0 .'86, resulting in a resolving power of $\lambda / \Delta \lambda \approx 55,000$, with a wavelength coverage of $\sim 3800-8000 \AA$. The iodine gas absorption cell was used to superimpose a dense forest of $\mathrm{I}_{2}$ lines on the stellar spectrum and establish an accurate wavelength fiducial (see Marcy \& Butler 1992). Relative RVs in the solar system barycentric frame were derived as described by Butler et al. (1996), incorporating full modeling of the spatial and temporal variations of the instrumental profile. The final RV data and their errors are listed in Table 1. The folded data, with our best fit (see Section 4) superimposed, are plotted in Figure 2. 


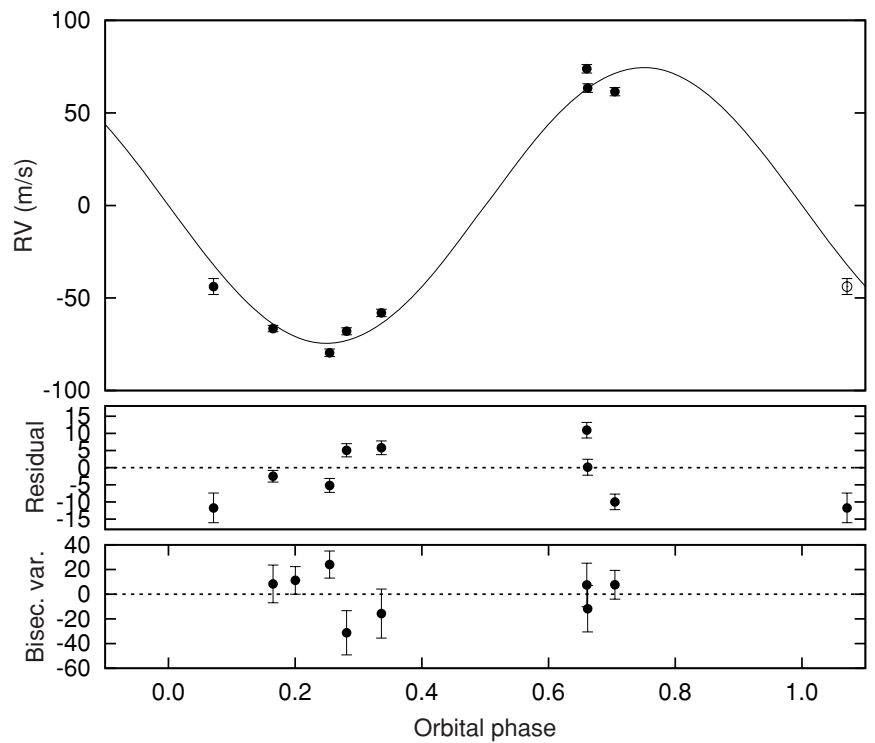

Figure 2. Top: RV measurements from Keck for HAT-P-10, along with an orbital fit, shown as a function of orbital phase, using our best-fit period (see Section 4). Zero phase is defined by the transit midpoint. The center-of-mass velocity has been subtracted. Middle: phased residuals after subtracting the orbital fit (also see Section 4). The rms variation of the residuals is about $4.2 \mathrm{~m} \mathrm{~s}^{-1}$. Bottom: Bisector spans for seven of the eight iodine spectra plus the iodine-free template spectrum (Section 4.2). The mean value has been subtracted. Note the different vertical scales of the panels.

\subsection{Photometric Follow-Up Observations}

We observed two complete transit events of HAT-P-10 on the night of 2008 September 19/20 MST and October $30 / 31$ MST with the KeplerCam CCD on the FLWO $1.2 \mathrm{~m}$ telescope. On September 19,278 frames were acquired with a cadence of $74 \mathrm{~s}$ (60 s of exposure time) in Sloan $z$ band, while on October 30, we gathered 405 frames with a cadence of nearly $60 \mathrm{~s}$ (40 s of exposure time) in Sloan $i$ band. The reduction of the images was performed as follows. After bias and flat calibration, we derived an initial second-order astrometrical transformation between the $\sim 110$ brightest stars and the 2MASS catalog, as described in Pál \& Bakos (2006), yielding a residual of $\sim 0.3-0.4$ pixels. In order to avoid systematic errors resulting from the proper motion of the stars, we generated a new catalog. This catalog was based on the detected stellar centroids, the coordinates of which were transformed to the same reference system using the initial astrometrical solutions, and then averaged using $3 \sigma$ rejection. Using this new catalog as reference, the final astrometrical solution was derived for each frame, yielding a residual of $\sim 0.02-0.04$ pixels. The primary reason for precise astrometry is to minimize the photometric errors that would originate from the centroiding errors for the individual stars on each frame. Instead, aperture photometry was performed on the above fixed positions, using a series of apertures with radii of $6.0,7.5,9.0$, and 10.5 pixels. The instrumental magnitude transformation was also done in two steps: first, all magnitude values were transformed to the photometric reference frame (selected to be the sharpest image), using the individual Poisson noise error estimations as weights. In the second step, the magnitude fit was repeated using the mean individual light-curve magnitudes as reference and the rms of these light curves as weights. In both of the magnitude transformations, we excluded from the fit the target star itself and the $3 \sigma$ outliers. We performed EPD against trends simultaneously with the light-curve modeling (for more details, see Section 4). From the series of apertures we chose

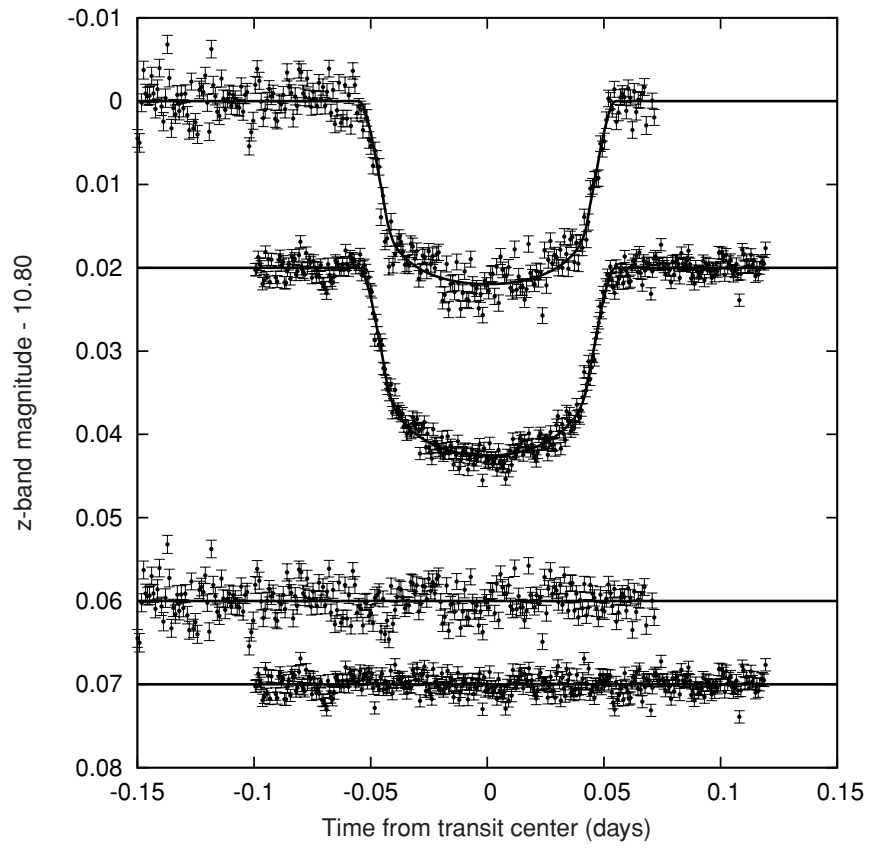

Figure 3. Unbinned instrumental Sloan $z$-band and $i$-band transit light curves, acquired with KeplerCam at the FLWO $1.2 \mathrm{~m}$ telescope on 2008 September 19 MST (upper plot) and 2008 October 30 MST (lower plot). Superimposed are the best-fit transit model light curves. In the bottom of the figure we show the residuals from the fit. The error bars represent the photon and background shot noise plus the readout noise.

Table 2

Photometry Follow-Up of HAT-P-10

\begin{tabular}{lrccc}
\hline \hline BJD & Magnitude $^{\mathrm{a}}$ & $\sigma_{\text {Mag }}$ & Magnitude (original) & Filter \\
\hline 2454729.73738 & -0.00128 & 0.00117 & 8.89747 & $z$ \\
2454729.73825 & 0.00243 & 0.00108 & 8.90114 & $z$ \\
2454729.73909 & -0.00384 & 0.00115 & 8.89491 & $z$ \\
2454729.73996 & 0.00112 & 0.00113 & 8.89988 & $z$ \\
2454770.75477 & 0.00023 & 0.00074 & 9.06540 & $i$ \\
2454770.75541 & 0.00022 & 0.00074 & 9.06546 & $i$ \\
2454770.75602 & -0.00127 & 0.00073 & 9.06405 & $i$ \\
2454770.75665 & -0.00040 & 0.00074 & 9.06485 & $i$ \\
\hline
\end{tabular}

Notes.

a The out-of-transit level has been subtracted. These magnitudes have been subjected to reconstructive EPD procedure, carried out simultaneously with the transit fit.

(This table is available in its entirety in a machine-readable form in the online journal. A portion is shown here for guidance regarding its form and content.)

the one with a radius of 7.5 pixels; this aperture yielded the smallest fit rms for both of the transit observations. This aperture falls in the middle of the aperture series, confirming the plausible selection for the apertures. The final light curves are shown in the upper plot of Figure 3, superimposed with our best-fit transit light-curve models (see also Section 4). Table 2 lists the individual magnitudes plotted in Figure 3.

\section{ANALYSIS}

In this section, we briefly describe our analysis yielding the orbital, planetary, and stellar parameters of the HAT-P-10 system.

\subsection{Planetary, Orbital, and Stellar Parameters}

First, using the template spectrum obtained by the Keck/HIRES instrument, we derived the stellar atmospheric 
parameters. We used the Spectroscopy Made Easy (SME) package of Valenti \& Piskunov (1996), which yielded the following values and uncertainties (which we have conservatively increased, based on past experience): $T_{\text {eff }}=4980 \pm 60 \mathrm{~K}$, $\log g_{\star}=4.5 \pm 0.1(\mathrm{cgs}),[\mathrm{Fe} / \mathrm{H}]=0.13 \pm 0.08$, and $v \sin i=$ $0.5 \pm 0.2 \mathrm{~km} \mathrm{~s}^{-1}$.

In modeling both the HATNet and the follow-up transit light curves, we used the quadratic limb darkening formalism of Mandel \& Agol (2002). The limb darkening coefficients corresponding to the above stellar atmospheric parameters were interpolated using the tables provided by Claret (2004). The coefficients we derived for $I, z$, and $i$ photometric passbands were $\gamma_{1(I)}=0.3806, \gamma_{2(I)}=0.2535, \gamma_{1(z)}=0.3214, \gamma_{2(z)}=0.2693$, $\gamma_{1(i)}=0.4104$, and $\gamma_{2(i)}=0.2457$.

Following this, a joint fit was done using all of the available data, including the HATNet light curve, the follow-up lightcurve, and the RV measurements. Throughout the analysis, we refer to the transit event observed on 2008 September 19-20 as $N_{\text {tr }}=0$.

We adjusted the following parameters: $T_{\mathrm{c},-290}$, the time of first transit center in the HATNet campaign; $T_{\mathrm{c},+11}$, the time of the transit center on October 30/31; $K$, the RV semiamplitude; $k=e \cos \omega$ and $h=e \sin \omega$, the Lagrangian orbital elements related to the eccentricity and argument of periastron; $p \equiv$ $R_{\mathrm{p}} / R_{\star}$, the fractional planetary radius; $b^{2}$, the square of the impact parameter; the quantity $\zeta / R_{\star}$, which is related to the transit duration $T_{\text {dur }}$ as $\left(\zeta / R_{\star}\right)^{-1}=T_{\text {dur }} / 2$; and $M_{0}$ and $M_{1}$, the out-of-transit instrumental magnitudes of the HATNet and FLWO/KeplerCam light curves. As noted by Bakos et al. (2007b), the quantity $\zeta / R_{\star}$ shows only a small correlation with the other light-curve parameters $\left(R_{\mathrm{p}} / R_{\star}, b^{2}\right)$, which makes it a good parameter to use. For eccentric orbits, $\zeta / R_{\star}$ is related to the normalized semimajor axis $a / R_{\star}$ as $\zeta / R_{\star}=$ $(2 \pi / P)\left(a / R_{\star}\right) \sqrt{1-e^{2}}\left(1-b^{2}\right)^{-1 / 2}(1+h)^{-1}$. To find the best-fit values and the uncertainties, we utilized the method of Markov chain Monte Carlo (MCMC; Ford 2006) which provides the a posteriori distribution of the adjusted parameters.

The values and uncertainties of the $k$ and $h$ orbital elements were found to be consistent with zero within nearly $1 \sigma$, namely, $k=-0.09 \pm 0.11$ and $h=-0.07 \pm 0.06$. Therefore, we conclude that the observations are consistent with a circular planetary orbit, and we repeated the fit by fixing the eccentricity to zero.

The key results for the simultaneous fit are reported in Table 4 . In addition, the fit yielded $T_{\mathrm{c},-290}=2453650.38936 \pm 0.00190$ (BJD), $T_{\mathrm{c}, 11}=2454770.85425 \pm 0.00016$ (BJD). These were used to derive the epoch and the period as shown in Pál et al. (2008b). The jitter in the RVs that was needed to reconcile the reduced $\chi^{2}$ of the fit with unity was $4.2 \mathrm{~m} \mathrm{~s}^{-1}$. The error bars in Figure 2 (top and middle panels) have been inflated accordingly.

The results of the joint fit together with the initial results from spectroscopy enable us to refine the parameters of the star. As described by Sozzetti et al. (2007) and Torres et al. (2008), $a / R_{\star}$ is a better luminosity indicator than the spectroscopic value of $\log g_{\star}$ since stellar surface gravity has only a subtle effect on the line profiles. Therefore, we used the values of $T_{\text {eff }}$ and $[\mathrm{Fe} / \mathrm{H}]$ from the initial SME analysis, together with the distribution of $a / R_{\star}$ to estimate the stellar properties from comparison with the Yonsei-Yale $\left(\mathrm{Y}^{2}\right)$ stellar evolution models by Yi et al. (2001) and Demarque et al. (2004). Using the relation between $a / R_{\star}$ and $\zeta / R_{\star}$, we derive the a posteriori distribution for the former one, and used the derived stellar density as an input for the stellar evolution models in order to have an a
Table 3

Stellar Parameters for HAT-P-10

\begin{tabular}{lcc}
\hline \hline Parameter & Value & Source \\
\hline$T_{\text {eff }}(\mathrm{K})$ & $4980 \pm 60$ & $\mathrm{SME}^{\mathrm{a}}$ \\
{$[\mathrm{Fe} / \mathrm{H}]$} & $0.13 \pm 0.08$ & $\mathrm{SME}$ \\
$v \sin i\left(\mathrm{~km} \mathrm{~s}^{-1}\right)$ & $0.5 \pm 0.2$ & $\mathrm{SME}$ \\
$M_{\star}\left(M_{\odot}\right)$ & $0.83 \pm 0.03$ & $\mathrm{Y}^{2}+\mathrm{LC}+\mathrm{SME}$ \\
$R_{\star}\left(R_{\odot}\right)$ & $0.79 \pm 0.02$ & $\mathrm{Y}^{2}+\mathrm{LC}+\mathrm{SME}$ \\
$\log g_{\star}(\mathrm{cgs})$ & $4.56 \pm 0.02$ & $\mathrm{Y}^{2}+\mathrm{LC}+\mathrm{SME}$ \\
$L_{\star}\left(L_{\odot}\right)$ & $0.34 \pm 0.03$ & $\mathrm{Y}^{2}+\mathrm{LC}+\mathrm{SME}$ \\
$M_{V}(\mathrm{mag})$ & $6.18 \pm 0.10$ & $\mathrm{Y}^{2}+\mathrm{LC}+\mathrm{SME}$ \\
Age $(\mathrm{Gyr})$ & $7.9 \pm 3.8$ & $\mathrm{Y}^{2}+\mathrm{LC}+\mathrm{SME}$ \\
Distance $(\mathrm{pc})$ & $121.7 \pm 4.2$ & $\mathrm{Y}^{2}+\mathrm{LC}+\mathrm{SME}$ \\
\hline
\end{tabular}

Notes.

a SME: "Spectroscopy Made Easy" package for analysis of high-resolution spectra (Valenti \& Piskunov 1996). See the text.

b $\mathrm{Y}^{2}+\mathrm{LC}+\mathrm{SME}$ : Yonsei-Yale isochrones (Yi et al. 2001), light-curve parameters, and SME results.

posteriori distribution for the stellar parameters (see Pál et al. 2008a, 2008b, for more details). Since the mass and radius (and their respective distributions) of the star are known, it is straightforward to obtain the surface gravity and its uncertainty together. The derived surface gravity is $\log g_{\star}=4.56 \pm 0.02$. Since the surface gravity from the initial SME analysis agrees well with the one derived above, we accept the latter as final value (listed together with other parameters in Table 3).

The stellar evolution modeling also yields the absolute magnitudes and colors in various photometric passbands. The derived $V-I$ color of the star is $(V-I)_{\mathrm{YY}}=0.92 \pm 0.02$, slightly smaller than the color from the The Amateur Sky Survey (TASS) catalog (Droege et al. 2006), namely $(V-I)_{\text {TASS }}=1.09 \pm 0.11$. Since this excess could be due to interstellar reddening, we used the 2MASS $J$ magnitude to estimate the distance. The observed $J$-band magnitude of HAT-P-10, after conversion to the same system used by the (Yi et al. 2001) isochrones, is $J=10.020 \pm 0.021$ while the stellar modeling gives $M_{J}=4.530 \pm 0.087$, which lead to a distance modulus for the star of $J-M_{J}=5.489 \pm 0.089$, corresponding to a distance of $d=121.7 \pm 4.2 \mathrm{pc}$.

The planetary parameters and their uncertainties can be derived by direct combination of the a posteriori distributions of the light-curve, RV, and stellar parameters. We find that the mass of the planet is $M_{p}=0.487 \pm 0.018 M_{\mathrm{J}}$, the radius is $R_{p}=$ $1.005_{-0.027}^{+0.032} R_{\mathrm{J}}$, and its density is $\rho_{p}=0.594 \pm 0.052 \mathrm{~g} \mathrm{~cm}^{-3}$. The planetary parameters are summarized at the bottom of Table 4.

\subsection{Excluding Blend Scenarios}

Following Torres et al. (2007), we explored the possibility that the measured RVs are not real, but are instead caused by distortions in the spectral line profiles due to contamination from a nearby unresolved eclipsing binary. A bisector analysis based on the Keck spectra was done as described in Section 5 of Bakos et al. (2007a).

The first spectrum in Table 1 was taken through clouds, at high-air mass, and near full moon, and is visibly contaminated by another set of lines at the expected velocity separation of about $-25 \mathrm{~km} \mathrm{~s}^{-1}$ due to the moonlight. The line bisector is highly sensitive to any distortions in the line profiles, and is thus seriously affected in this case. The measured velocity, on the other hand, is affected only at a much lower level 

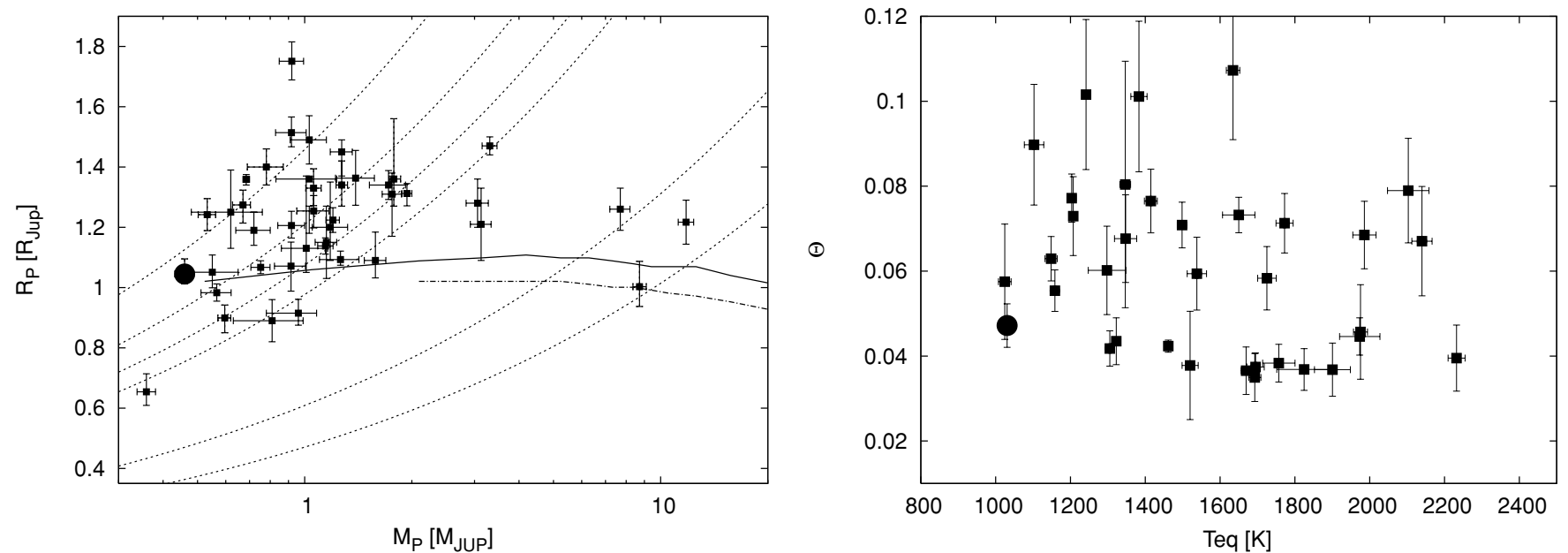

Figure 4. Left: mass-radius diagram of TEPs. HAT-P-10b is shown as a large filled circle on the left. Overlaid are Baraffe et al. (2003) zero insolation planetary isochrones for ages of $0.5 \mathrm{Gyr}$ (upper, solid line) and $5 \mathrm{Gyr}$ (lower dash-dotted line), respectively, as well as isodensity lines for $0.4,0.7,1.0,1.33,5.5$, and $11.9 \mathrm{~g} \mathrm{~cm}^{-3}$ (dashed lines). Right: equilibrium temperature vs. Safronov number (Hansen \& Barman 2007).

Table 4

Orbital and Planetary Parameters

\begin{tabular}{|c|c|}
\hline Parameter & Value \\
\hline \multicolumn{2}{|c|}{ Light-curve parameters } \\
\hline$P$ (days) & $3.7224747 \pm 0.0000065$ \\
\hline$E(\mathrm{BJD})$ & $2454759.68683 \pm 0.00016$ \\
\hline$T_{14}$ (days) $^{\mathrm{a}}$ & $0.1090 \pm 0.0008$ \\
\hline$T_{12}=T_{34}(\text { days })^{\mathrm{a}}$ & $0.0137 \pm 0.0007$ \\
\hline$b^{2}$ & $0.080 \pm 0.042$ \\
\hline$\zeta / R_{\star}\left(\right.$ day $\left.^{-1}\right)$ & $20.72 \pm 0.14$ \\
\hline$a / R_{\star}$ & $11.87 \pm 0.27$ \\
\hline$R_{p} / R_{\star}$ & $0.1315 \pm 0.0010$ \\
\hline$b \equiv a \cos i / R_{\star}$ & $0.282_{-0.095}^{+0.067}$ \\
\hline$i(\mathrm{deg})$ & $88.6_{-0.4}^{+0.5}$ \\
\hline \multicolumn{2}{|c|}{ Spectroscopic parameters } \\
\hline$K\left(\mathrm{~m} \mathrm{~s}^{-1}\right)$ & $74.5 \pm 1.8$ \\
\hline$\gamma\left(\mathrm{km} \mathrm{s}^{-1}\right)^{\mathrm{b}}$ & $3.95 \pm 0.43$ \\
\hline$\gamma_{\text {Keck }}\left(\mathrm{m} \mathrm{s}^{-1}\right)^{\mathrm{c}}$ & $79.6 \pm 1.7$ \\
\hline$e$ & 0 (adopted) \\
\hline \multicolumn{2}{|c|}{ Planetary parameters } \\
\hline$M_{p}\left(M_{\mathrm{J}}\right)$ & $0.487 \pm 0.018$ \\
\hline$R_{p}\left(R_{\mathrm{J}}\right)$ & $1.005_{-0.027}^{+0.032}$ \\
\hline$C\left(M_{p}, R_{p}\right)$ & 0.206 \\
\hline$\rho_{p}\left(\mathrm{~g} \mathrm{~cm}^{-3}\right)$ & $0.594 \pm 0.052$ \\
\hline$a(\mathrm{AU})$ & $0.0435 \pm 0.0006$ \\
\hline $\log g_{p}(\mathrm{cgs})$ & $3.08 \pm 0.03$ \\
\hline$T_{\text {eq }}(\mathrm{K})$ & $1020 \pm 17$ \\
\hline$\Theta$ & $0.053 \pm 0.002$ \\
\hline
\end{tabular}

Notes.

${ }^{\mathrm{a}} T_{14}$ : total transit duration, time between first and last contact; $T_{12}=T_{34}$ : ingress/egress time, time between first and second, or third and fourth contact.

b The systemic velocity on an absolute scale, based on the DS observations.

c The RV zero point of the Keck data in the fit on a relative scale.

that we consider tolerable (although it still shows the largest negative residual). We have thus chosen not to use the bisector span from this spectrum, but to keep the velocity. Among the remaining bisector spans, we detect no variation in excess of the measurement errors (see Figure 2, bottom panel). The correlation between the RV and the bisector variations is insignificant. Therefore, we conclude that the velocity variations are real, and that the star is orbited by a close-in giant planet.

\section{DISCUSSION}

It is interesting to compare the properties of HAT-P-10b with the other known TEPs so as to place it in a broader context. This planet falls at the low-mass end of the current distribution, as shown in Figure 4 (left panel). There are only three TEPs known with smaller masses; these are HD 149026b (Sato et al. 2005), GJ436b (Butler et al. 2004), and HAT-P-11b (Bakos et al. 2009), where the first two have been discovered by RV searches, and the latter by the HATNet transit search. In Figure 4, we overplot Baraffe et al. (2003) planetary isochrones, which indicate that the radius of HAT-P-10b is broadly consistent with these models.

We compared our mass and radius to theoretical estimates of Liu et al. (2008) for a $0.5 M_{\mathrm{J}}$ body at various orbital distances from a $\mathrm{G} 2 \mathrm{~V}$ star. We note that the equivalent semimajor axis (with the same incident flux) of HAT-P-10b around a solartype star is $a_{\text {rel }}=0.076$. It is also noteworthy that when a detailed comparison is done, the effects of the environment on the planetary properties are not as simple as scaling the integrated stellar flux, since the detailed spectrum of the star (e.g., UV flux) may also be important.

Based on the models presented by Liu et al. (2008), for $\dot{E}_{\mathrm{h}} / \dot{E}_{\text {ins }}=10^{-6}$ the equilibrium radius of HAT-P-10b would be $\sim 1.15 R_{\mathrm{J}}$, where $E_{\mathrm{h}}$ is the energy per unit time due to orbital tidal heating or similar internal heating, and $\dot{E}_{\text {ins }}$ is the energy received via insolation. For larger values of $\dot{E}_{\mathrm{h}} / \dot{E}_{\text {ins }}$ the expected radius is larger, and for smaller values it asymptotically converges to $1.15 R_{\mathrm{J}}$. This makes us conclude that a small core is required so that the model values match the observed $1.00 R_{\mathrm{J}}$ radius of HAT-P-10b. This need for a core is consistent with the core-mass-stellar metallicity relation proposed by Guillot et al. (1996) and Burrows et al. (2007). We obtain similar results if we compare the observations to models of Fortney et al. (2008). Namely, the current mass, radius, and insolation of HAT-P-10b are consistent with both a $300 \mathrm{Myr}$ model with a $40 M_{\oplus}$ core mass, and also with a 4.5 Gyr almost pure hydrogen and helium model with a small $\sim 10 M_{\oplus}$ core.

The radiation that HAT-P-10b receives from its host star is $\sim 2.56 \times 10^{8} \mathrm{erg} \mathrm{s}^{-1} \mathrm{~cm}^{-2}$. With the definitions of Fortney et al. (2008), HAT-P-10b belongs to the pL class of planets. There is only one transiting planet that has a lower mean incident 
flux: HD 17156b (Barbieri et al. 2007). However, this planet orbits on a highly eccentric orbit, and the incident flux increases to over $10^{9} \mathrm{erg} \mathrm{s}^{-1} \mathrm{~cm}^{-2}$ at periastron, i.e., four times that of HAT-P-10b.

The other planet with a similarly low incident flux is OGLETR-111b (Udalski et al. 2002; Pont et al. 2004), orbiting an $I=15.55 \mathrm{mag}$ star with $T_{\text {eff }}=5040 \mathrm{~K}$ (Santos et al. 2006). HAT-P-10b appears to be a nearby analog of OGLETR-111b in many respects, since their parameters are very similar (parentheses show those of OGLE-TR-111); the period is 3.7225 days (4.01 days), the stellar mass is $0.83 M_{\odot}\left(0.85 M_{\odot}\right)$, the stellar radius is $0.79 R_{\odot}\left(0.83 R_{\odot}\right)$, the luminosity is $0.36 L_{\odot}$ $\left(0.4 L_{\odot}\right)$, the metallicity is $0.13 \pm 0.08(0.19 \pm 0.07)$, and the planetary radius is $1.00 M_{\mathrm{J}}\left(1.05 M_{\mathrm{J}}\right)$. Interestingly, even the impact parameter of their transits is similar. There is a slight difference in their masses, with HAT-P-10b being smaller $\left(0.49 \pm 0.02 M_{\mathrm{J}}\right.$ vs. $\left.0.55 \pm 0.1 M_{\mathrm{J}}\right)$. One crucial difference between the two systems is that HAT-P-10 is 10 times closer to us, being at $121.7 \pm 4.2 \mathrm{pc}$ versus $1500 \mathrm{pc}$ for OGLE-TR-111, and is more than 4 mag brighter, thus enabling more detailed follow-up in the near future.

Another interesting observational fact is that the $\Theta=0.053 \pm$ 0.002 Safronov number of HAT-P-10b falls fairly close to the dividing line between the proposed Class I and Class II planets (Hansen \& Barman 2007). At the low end of the equilibrium temperature range of the plot (excluding GJ436b), HAT-P-10b seems to be at a point where the two distributions overlap (see Figure 4, right panel). Finally, we note that HAT-P-10b strengthens the orbital period versus surface gravity relation (Southworth et al. 2007), falling almost exactly on the linear fit between these two quantities (Torres et al. 2008).

HATNet operations have been funded by NASA grants NNG04GN74G, NNX08AF23G, and SAO IR\&D grants. The efforts of G.Á.B. and J.A.J. were supported by the Postdoctoral Fellowship of the NSF Astronomy and Astrophysics Program (AST-0702843 and AST-0702821, respectively). We also acknowledge partial support from the Kepler Mission under NASA Cooperative Agreement NCC2-1390 (PI: D.W.L.). G.K. thanks the Hungarian Scientific Research Foundation (OTKA) for support through grant K-60750. This research has made use of Keck telescope time granted through NOAO (program A285Hr) and NASA (N128Hr).

\section{REFERENCES}

Bakos, G. Á., Lázár, J., Papp, I., Sári, P., \& Green, E. M. 2002, PASP, 114, 974 Bakos, G. Á., Noyes, R. W., Kovács, G., Stanek, K. Z., Sasselov, D. D., \& Domsa, I. 2004, PASP, 116, 266

Bakos, G. Á., et al. 2007a, ApJ, 670, 826

Bakos, G. Á., et al. 2007b, ApJ, 671, L173
Bakos, G. Á., et al. 2009, ApJ, submitted (arXiv:0901.0282)

Baraffe, I., Chabrier, G., Barman, T. S., Allard, F., \& Hauschildt, P. H. 2003, A\&A, 402, 701

Barbieri, M., et al. 2007, A\&A, 476, L13

Brown, T. M., \& Charbonneau, D. 2000, in ASP Conf. Ser. 219, Disks, Planetesimals, and Planets, ed. F. Garzón et al. (San Francisco, CA: ASP), 584

Burrows, A., Hubeny, I., Budaj, J., \& Hubbard, W. B. 2007, ApJ, 661, 502

Burrows, A., Sudarsky, D., \& Hubeny, I. 2006, ApJ, 650, 1140

Butler, R. P., Vogt, S. S., Marcy, G. W., Fischer, D. A., Wright, J. T., Henry, G. W., Laughlin, G., \& Lissauer, J. J. 2004, ApJ, 617, 580

Butler, R. P., et al. 1996, PASP, 108, 500

Chabrier, G., Barman, T., Baraffe, I., Allard, F., \& Hauschildt, P. H. 2004, ApJ, 603, L53

Claret, A. 2004, A\&A, 428, 1001

Cutri, R. M., et al. 2003, The IRSA 2MASS All-Sky Point Source Catalog, NASA/IPAC Infrared Science Archive

Demarque, et al. 2004, ApJ, 155, 667

Droege, T. F., Richmond, M. W., \& Sallman, M. 2006, PASP, 118, 1666

Dunham, E. W., Mandushev, G. I., Taylor, B. W., \& Oetiker, B. 2004, PASP, 116, 1072

Ford, E. 2006, ApJ, 642, 505

Fortney, J. J., Lodders, K., Marley, M. S., \& Freedman, R. S. 2008, ApJ, 678, 1419

Fortney, J. J., Marley, M. S., \& Barnes, J. W. 2007, ApJ, 659, 1661

Gillon, M., et al. 2007, A\&A, 472, L13

Guillot, T., Burrows, A., Hubbard, W. B., Lunine, J. I., \& Saumon, D. 1996, ApJ, 459, L35

Hansen, B. M. S., \& Barman, T. 2007, ApJ, 671, 861

Johns-Krull, C. M., et al. 2008, ApJ, 677, 657

Konacki, M., Torres, G., Jha, S., \& Sasselov, D. D. 2003, Nature, 421, 507

Kovács, G., Bakos, G. Á., \& Noyes, R. W. 2005, MNRAS, 356, 557

Kovács, G., Zucker, S., \& Mazeh, T. 2002, A\&A, 391, 369

Latham, D. W. 1992, in IAU Coll. 135, in ASP Conf. Ser. 32, Complementary Approaches to Double and Multiple Star Research, ed. H. A. McAlister \& W. I. Hartkopf (San Francisco, CA: ASP), 110

Liu, X., Burrows, A., \& Ibgui, L. 2008, arXiv: 0805.1733

Mandel, K., \& Agol, E. 2002, ApJ, 580, L171

Marcy, G. W., \& Butler, R. P. 1992, PASP, 104, 270

McCullough, P. R., Stys, J. E., Valenti, J. A., Fleming, S. W., Janes, K. A., \& Heasley, J. N. 2005, PASP, 117, 783

Pál, A., \& Bakos, G. Á. 2006, PASP, 118, 1474

Pál, A., Bakos, G. Á., Noyes, R. W., \& Torres, G. 2008a, in IAU Symp. 253, Transiting Planets, ed. F. Pont, 428

Pál, A., et al. 2008b, ApJ, 680, 1450

Pollacco, D. L., et al. 2006, PASP, 118, 1407

Pont, F., Bouchy, F., Queloz, D., Santos, N. C., Melo, C., Mayor, M., \& Udry, S. 2004, A\&A, 426, L15

Santos, N. C., et al. 2006, A\&A, 450, 825

Sato, B., et al. 2005, ApJ, 633, 465

Southworth, J., Wheatley, P. J., \& Sams, G. 2007, MNRAS, 379, 11

Sozzetti, A., et al. 2007, ApJ, 664, 1190

Torres, G., Boden, A. F., Latham, D. W., Pan, M., \& Stefanik, R. P. 2002, AJ, 124,1716

Torres, G., Winn, J. N., \& Holman, M. J. 2008, ApJ, 677, 1324

Torres, G., et al. 2007, ApJ, 666, 121

Udalski, A., et al. 2002, Acta Astron., 52, 1

Udalski, A., et al. 2008, A\&A, 482, 299

Valenti, J. A., \& Piskunov, N. 1996, A\&AS, 118, 595

Vogt, S. S., et al. 1994, Proc. SPIE, 2198, 362

West, R., et al. 2008, A\&A, submitted, (arXiv:0809.4597)

Yi, S. K., et al. 2001, ApJS, 136, 417 